reticulocyte lysates, which lack $R$ Nase III, is so potent that this small region of poliovirus ds RNA might be sufficient to produce the inhibitory effects seen in vivo.

The mechanism of action of ds RNA in reticulocyte lysates is the subject of another recent paper in Proc. natn. Acad. Sci. U.S.A. Benzard and London $(71,2863-2866 ; 1974)$ have shown that the inhibitory effect can be prevented or reversed by addition of the initiation factor $\mathbf{M}_{3}$. Preparations of initiation factors $M_{1}$ and $M_{2}$ (which are responsible for the binding of initiator tRNA) and ribosome dissociation factor are ineffective. These observations are in agreement with similar experiments by Kaempfer and Kaufman (Proc. natn. Acad. Sci. U.S.A., 70, 1222-1226; 1973) who showed that ds RNA inactivates reticulocyte initiation factor $\mathrm{IF}_{3}$, but both reports conflict with those of Darnbrough, Hunt and Jackson (Biochem. biophys. Res. Commun., 48, $1556-1564$; 1972) who found that ds RNA inhibits the formation of the complex between $40 \mathrm{~S}$ ribosomal subunits and initiator tRNA. At present it is difficult to resolve this apparent contradiction. But it seems that very small amounts of ds RNA inhibit initiation by sequestering a catalytic site which normally recognises some structural feature in another RNA molecule. Such a structure could be present in mRNA, rRNA, initiator tRNA or perhaps even the small RNA molecule discussed earlier.

\section{Archaeopteryx flies again}

from Barry Cox

Archaeopteryx is probably the best example of an evolutionary intermediate between two major groups of animal. It is normally accepted that it is not far removed from the arboreal reptilian stock from which birds evolved. Though its skeleton is not greatly modified for flight, the elongated feathers of Archaeopteryx strongly suggest that flight of some kind had already evolved.

Ostrom (Q. Rev. Biol., 49, 27; 1974) has now questioned the idea that bird flight evolved in an arboreal environment. He starts from the observation that Archaeopteryx and the smaller cursorial dinosaurs such as Ornitholestes show so many characteristics in common that it would be surprising if their habits of life were totally different. He also states that the terminal phalanges of the foot of Archaeopteryx are relatively straight and robust, like those of cursorial birds but unlike those of perching birds, which are strongly curved.
It has long been noted that, surprisingly, the manus of Archaeopteryx has three long, unfused clawed fingers, and these have been interpreted as adaptations to climbing in trees. Ostrom shows that the hand, and the whole pectoral skeleton, of Archaeopteryx are very similar to those of such dinosaurs as Ornitholestes, and that the claws were long, curved and pointed-more suitable, he thinks, to a prey-grasping function that to climbing. $\mathrm{He}$ also notes that neither the pectoral skeleton nor the long many-feathered tail of Archaeopteryx suggests that it could have been an active filer.

Ostrom's view is that Archaeopteryx was a bipedal fast-running form which lived on "relatively small animals, most probably large insects, and perhaps small lizards and mammals". He believes that feathers evolved to aid in thermal insulation, and that their elaboration on the forelimb was originally to increase its efficiency as a preycatching appendage, the two arms "becoming a trapping device or net with which to corral or surround small prey so that they could be more easily grasped in the mouth or hand". True flight, Ostrom believes, evolved from flapping, leaping attacks on such prey.

Long-established theories are often found to be based on deductions which are today either discarded or less certain, and it is usually worthwhile to attempt a fundamental reappraisal. Ostram's work seems certain to provoke further discussion and criticismboth of the orthodox theory and of his own views. Walker (Nature, 237, 257; 1972) has already suggested that birds are more closely related to crocodiles than to theropod dinosaurs, but both Gingerich (Nature, 243, 70; 1973) and Bakker and Galton (Nature, 248, 168; 1974) have supported the link with dinosaurs-the latter even to the extent of including birds in a new class Dinosauria.

Some aspects of Ostrom's theories seem somewhat unlikely, at least at first glance. It is always heartening to the palaeontologist to be able to see similar adaptations in use today, but there are none such for Archaeopteryx's supposed insect-snaring spread of feathers. These would also have been of little use (and possibly a hindrance) in catching the small lizards and mammals that Ostrom proposes as alternative prey. His suggestion that the long, feathered tail was a braking mechanism seems equally unconvincing. Ostrom may well be right in suggesting that the ancestors of Archaeopteryx evolved bipedality as ground-running forms, like some modern lizards (in which bipedal and quadrupedal locomotion are not fundamentally different), and that they only evolved the extensive spread of feathers as a gliding adaptation after they had become arboreal forms. Nevertheless, the adoption of a dramatically different method of locomotion (flight) might well have followed from colonisation of a very different habitat, such as trees. The imperfection of Archaeopteryx as an arboreal gliding form and its retention of a grasping hand might then merely indicate a relatively recent adoption of this way of life, rather than disproving it.

Other interested parties are girding their loins for the fray, and it will be interesting to see whether Archaeopteryx ends up with its feet on the ground or its head in the air.

\section{Agricultural waste to supplement oil}

\section{from D. A. H. Taylor}

IN 1949 Dupont opened a plant at Niagara for the production of nylon intermediates from furfural. This has long been closed down, as it could not compete with petrochemical routes, but it may now be time to reconsider the position of furfural in the light of the present oil situation.

Furfural is obtained by acidic hydrolysis of pentosans, contained in agricultural waste. Almost any plant material may be used, including straw and sawdust, with an average yield of $5-10 \%$. Present sources include oat hulls, maize cobs, and bagasse, which is the residue after extracting sucrose from sugar cane. The potential supplies are large: it has been calculated that 2 million tons of furfural could be produced per annum in the United States from maize cobs alone (Dunlop and Peters, The Furans, 283, Reinhold, New York; 1953); the potential yield from sugar cane is roughly equal to that of sucrose, currently about 50 million tons $\mathrm{yr}^{-1}$. This may be compared with a world production in 1970 of approximately 40 million tons of primary petrochemicals (Imhausen, Chem. Ind., 1559; 1970.)

It is possible to make practically any petrochemicals one wishes from furfural, at a price. As the sources of furfural are usually of slight alternative value, the main expenses in production are plant construction, operating costs and handling and collection charges for the raw material. Its present uses are mainly as a solvent and in the synthesis of resins used to manufacture grinding wheels and brake linings. A major use is the production of resin for impregnating the glass fibre mats wrapped around steel pipelines to prevent corrosion, and currently the demand in connection with the construction of Arctic pipelines has raised the price of furfural delivered in Europe 